Review Article

\title{
The Impact of Natural Product Dietary Supplements on Patients with Gout: A Systematic Review and Meta-Analysis of Randomized Controlled Trials
}

\author{
Juan Yang, ${ }^{1,2}$ Guangxi Li $\mathbb{D}^{1,3,4}$ Donglin Xiong, ${ }^{2}$ Tony Y. Chon, ${ }^{1}$ and Brent A. Bauer $\mathbb{D}^{1}$ \\ ${ }^{1}$ Division of General Internal Medicine, Mayo Clinic, Rochester, MN 55905, USA \\ ${ }^{2}$ Department of Pain Medicine, Shenzhen Nanshan People's Hospital, Guangdong Medical University, Shenzhen, \\ Guangdong 518052, China \\ ${ }^{3}$ Guang'anmen Hospital, China Academy of Chinese Medical Sciences, Beijing 100053, China \\ ${ }^{4}$ Division of Pulmonary and Critical Care Medicine, Mayo Epidemiology and Translational Research in Intensive Care, Rochester, \\ MN 55905, USA
}

Correspondence should be addressed to Brent A. Bauer; bauer.brent@mayo.edu

Received 31 August 2019; Revised 19 November 2019; Accepted 27 December 2019; Published 25 January 2020

Guest Editor: Samuel Martins Silvestre

Copyright (c) 2020 Juan Yang et al. This is an open access article distributed under the Creative Commons Attribution License, which permits unrestricted use, distribution, and reproduction in any medium, provided the original work is properly cited.

Natural product dietary supplements (NPDS) are frequently used for the treatment of gout, but reliable efficacy and safety data are generally lacking or not well organized to guide clinical decision making. This review aims to explore the impacts of NPDS for patients with gout. An electronic literature search was conducted to retrieve data published in English language from databases from inception to August 14, 2019. Randomized controlled trials (RCTs) that compared NPDS with or without placebo, diet modification, conventional pharmaceutics, or the other Chinese medicine treatment for gout patients were included. Two authors screened the articles, extracted the data, and assessed the risk of bias of each included trial independently. Meta-analysis was performed using Review Manager version 5.3.5. Results. Nine RCTS were enrolled in this review. The methodological quality of the nine RCTs was poor. The study results showed that in the majority of trials, NPDS demonstrated some degree of therapeutic efficacy for joint swelling, pain, and activity limitation. In contradistinction, serum uric acid (SUA) level (SMD -1.80, 95\% CI: -4.45 to 0.86$)(p>0.05)$ and CRP levels $(N=232$; SMD, -0.26 ; $95 \% \mathrm{CI},-0.55$ to 0.04$)(p>0.05)$ did not improve significantly. The incidence of adverse events (AEs) was not lower in the participants treated with NPDS ( $N=750$; RR, 0.47; 95\% CI, 0.20-1.11) $(p>0.05)$. Conclusion. Current existing evidence is not sufficient to provide clinical guidance regarding the efficacy and safety of NPDS as a treatment for gout due to poor trial quality and lack of standardized evaluation criteria. Larger and more rigorously designed RCTs are needed in the future.

\section{Introduction}

Gout is a form of inflammatory arthritis resulting from the deposition of monosodium urate crystals in the synovial joints and soft tissues in the setting of hyperuricemia, i.e., elevated serum uric acid level (SUA $>6.8 \mathrm{mg} / \mathrm{dL}$ ) [1]. It is a chronic and often debilitating disease featured by recurrent swelling, redness and pain in one or multiple joints, which, if untreated or poorly controlled, can lead to limited musculoskeletal function, work-related disability, and significant morbidities such as hypertension, cardiovascular disease, chronic kidney disease, and poor health-related quality of life $[2,3]$. The prevalence of gout, as well as its social and economic burden, has been rising globally, especially for middle-aged and elderly men. In 2007-2008, the prevalence of gout among US adults (8.3 million individuals) was $3.9 \%$ [4], and the incidence has continued to rise, due in part to economic development, dietary habit changes, aging population, $l$, and increasing-associated comorbidities $[1,5]$.

Both American and European rheumatology society guidelines for gout management focus on controlling gout flares and long-term reduction in total uric acid levels $[6,7]$. 
Conventional medicines are limited in patients with gout because of interactions, inherent drug toxicity, and polypharmacy for patients with multiple comorbidities. Dietary therapy can lower uric acid levels, delay gout complications, and, in many cases, reduce or elimilate the need for conventional medications. Strong evidence suggests that diet is the most modifiable factor in gout management [8-10].

Natural product dietary supplements (NPDS), also named natural dietary supplements (NPS), are defined as systemically ingested, nonmineral, nonvitamin, and natural product-derived substances [11]. Previous studies have suggested that natural products including vegetables, nuts, legumes, fruits, and whole grains play an important role in the development of hyperuricemia and gout [12]. To date, no specific guidelines have recommended NPDS for gouty arthritis. Many publications have discussed the importance of natural compounds derived from animals, plants, and microbe sources for the treatment of human diseases [13]. Natural products are often regarded by consumers and patients as gentle and safe to human body because of their natural properties. In reality, the active ingredients of these natural products are compounds, which, if powerful enough to provide a beneficial effect, may also be powerful enough to cause adverse effects [14]. Natural products can regulate both the production and the excretion of uric acid, which may benefit the treatments of hyperuricemia, as reported in a previous review [15]. Several herbal compounds have been found to have antihyperuricemic effects in vitro and in vivo, suggesting they may play a role in the treatment of gout arthritis, while further research studies are needed to explore their potential action [16].

Standard epidemiology and treatment are required to further evaluate and verify the clinical efficacy and safety of the NPDS in gout treatment; therefore, providing valid and reliable data for accurate application of NPDS therapy are needed in the future [17].

Hence, our review aims to reveal some insights from previously published, randomized, controlled trials (RCTs) exploring the impacts of NPDS on gout patients and presents the evidence for natural dietary.

\section{Materials and Methods}

2.1. Search Strategy. A comprehensive electronic literature search was performed in the following databases from the database inception to August 14, 2019, limited to English language only, and excluding animal studies: Ovid MEDLINE (R) and Epub Ahead of Print, In-Process \& Other Non-Indexed Citations and Daily, Ovid Cochrane Central Register of Controlled Trials, Ovid Embase, Ovid Cochrane Database of Systematic Reviews, and Scopus. Search terms used were (natural product OR diet OR supplement OR remedy OR remedies OR medicine OR prescriptions OR preparations $\mathrm{OR}$ extract $\mathrm{OR}$ indigenous $\mathrm{OR}$ traditional $\mathrm{OR}$ alternative OR complementary OR primitive OR Chinese OR China OR Japan OR Kampo OR oriental OR Asian OR Korean OR native American OR Indian OR Hindu OR siddha OR Tibet OR Africa OR Brazil OR rongoa OR Ayurveda OR herb OR natural OR plant OR flower OR fruit
OR leaf OR leaves OR tea) AND (antigout OR anti-gout OR gout OR gouty OR hyperuricemia). All retrieved papers were manually scanned to identify further possible articles missed by electronic searching. Two reviewers screened the publications independently; any discrepancy between the two reviewers was resolved by consensus or by a discussion with a third reviewer if needed. The quality of RCTs was assessed using the Cochrane Collaboration Risk of Bias Assessment Tool [18]. The PRISMA flow chart is shown in Figure 1.

2.2. Participants. All subjects were aged 18 years or above diagnosed with gout based on the diagnostic criteria [6].

2.3. Interventions. All trials evaluating any natural product or natural compounds derived from animals, plants and microbe sources alone or in combination were included.

2.4. Comparators. All trials with any control groups such as no treatment/waiting list, sham therapy/placebo, nonpharmacological therapy (e.g., diet modification), and pharmacological therapy (e.g., allopurinol), alone or in combined therapies, were enrolled.

2.5. Outcomes. Outcome measures should include at least one or more of the following measurements: (1) pain relief evaluated by the visual analogue scale (VAS) or numeric rating scale (NRS); (2) joint function improvement by the NRS; (3) health-related quality of life by 36-Item Short-Form Health Survey (SF-36) and the Gout Assessment Questionnaire; (4) clinical efficacy identified by reduction in SUA levels; (5) inflammation markers by white blood cell (WBC) count, erythrocyte sedimentation rate (ESR), and C-reactive protein (CRP) level; and (6) safety was monitored by the reporting of adverse events (AEs).

2.6. Selected Trials. RCTs involving human participants were included in this review. Any study focused on the efficacy and safety of NPDS (single or compound) on gout was included. The abstract publication languages were restricted to English language only.

2.7. Data Extraction. Two review authors extracted relevant information independently from the retrieved trials. An Excel spreadsheet was designed to record descriptive data, the methodological quality of original studies, treatment regimen and duration, outcomes, efficacy, and AEs. The raw data such as means and standard deviations (SD) for continuous outcomes and event numbers or participants for dichotomous outcomes were extracted. Any disagreement between the two reviewers was resolved by a discussion between the two reviewers or referring to the original author.

2.8. Assessment of Risk of Bias in Included Studies. The potential bias of each trial was assessed using Cochrane Risk of Bias Tool for Randomized Controlled Trials [19] by two 


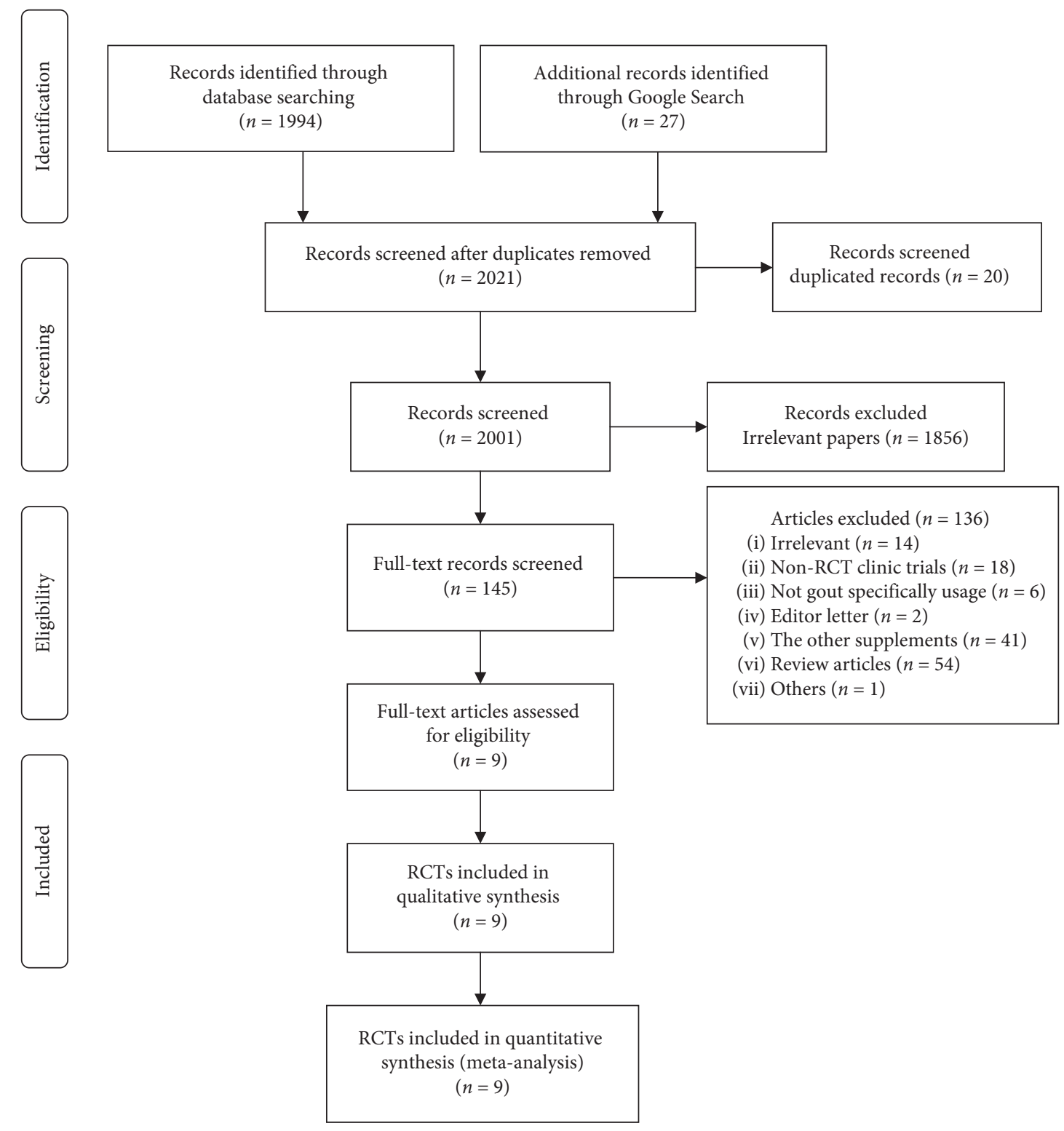

FIGURE 1: PRISMA flow chart.

reviewers independently in the following domains: risk of selection, reporting, performance, detection, attrition, and other sources of. Each domain was assessed as either "high," "low," or "unclear" bias. Any discrepancy was resolved by consensus or resort to a third review author.

2.9. Data Synthesis. Treatment efficacy was analyzed with the Cochrane Collaboration's statistical software, RevMan version 5.3.5 (Cochrane Collaboration, London, UK). Outcomes of continuous variables were presented as mean differences (MDs) between the observation and control groups with the corresponding 95\% confidence intervals (CIs). A metaanalysis was performed only if the studies were sufficiently homogeneous. Results of dichotomous data were presented as risk ratios (RRs) with the corresponding 95\% CIs. The random-effect model was applied, if $I^{2}>50 \%$ indicated significant heterogeneity among the studies. Subgroup analysis was performed according to the different NPDS interventions. The study number of each outcome was insufficient, so publication bias could not be analyzed with a funnel plot.

\section{Results}

3.1. Characteristics of the Trials. The literature search retrieved 2021 references, of which 20 were duplicates; 1856 articles were rejected on reading their titles and abstracts, and 145 articles were identified for detailed review. Finally, 9 articles were included (Figure 2).

A total of nine RCTs with 1156 participants were included in this review. Seven trials were conducted in China [20-26], 1 in the USA [27], and 1 in New Zealand [28]. All the studies were published from 2008 to 2019. Seven studies evaluated herbal therapies, with the remaining two evaluating skim milk and cherry extract. Six trials had two arms; 

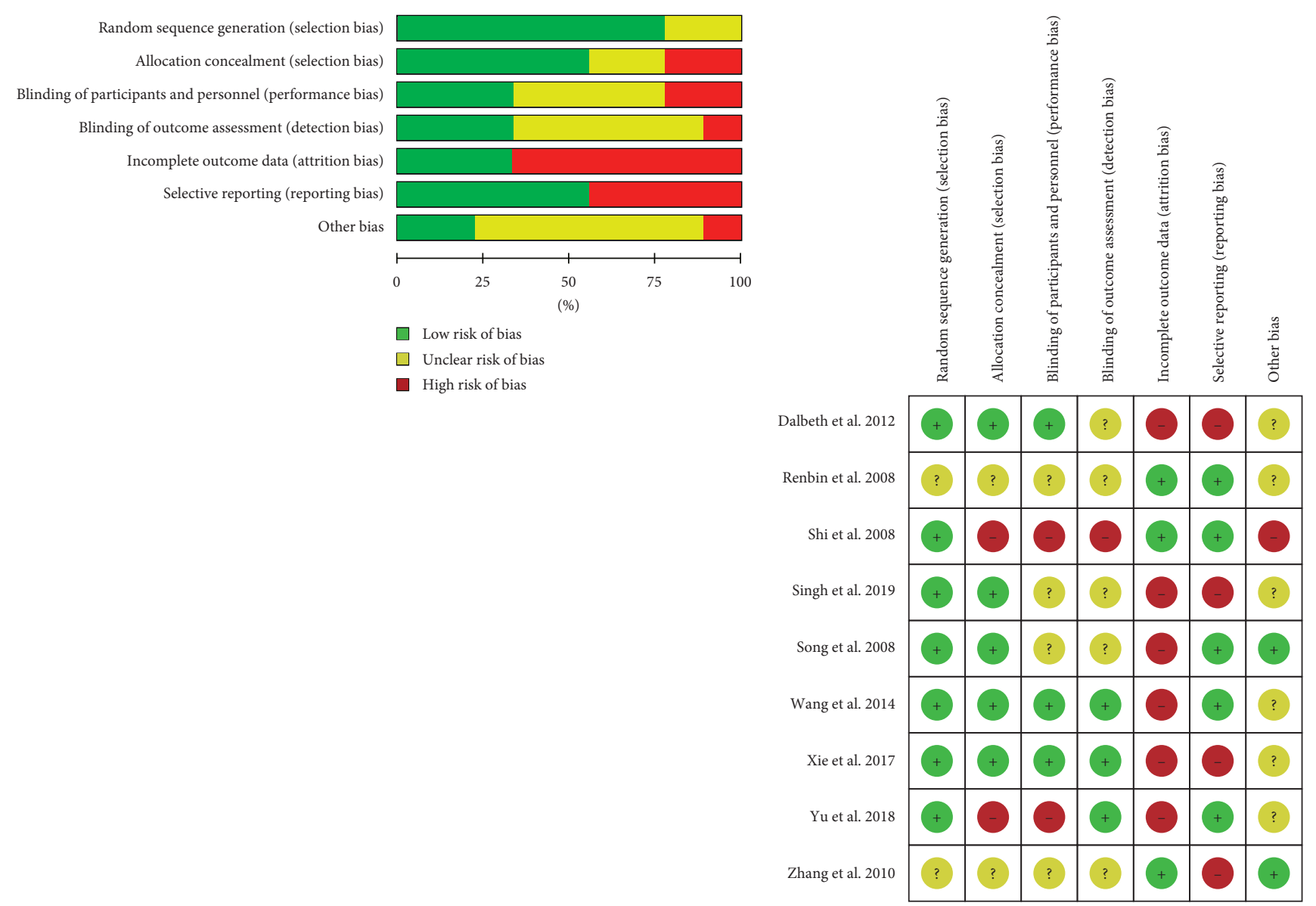

(a)

(b)

Figure 2: Risk of bias summary.

two trials had three arms; one trial had four arms. Five trials reported natural product formulation in decoction, two in capsules, and one in powder. Five trials recorded gout flareup; four trials did not record gout flare-up. Five trials reported no AEs; four trials reported AEs. Two trials assessed the compliance of patients (Table 1).

3.2. Methodological Quality. Seven of the nine RCTs had lower risk regarding randomization due to reported methods of sequence generation. Three trials used a random number table $[22,24,28]$, two trials referred to SAS software for setting the random variable seeds $[20,25]$, one adopted the random envelope method [21], and the other used online computer random number generator [27]. The other two trials had an unclear risk for random sequence generation due to lack of detail $[23,26]$. Two trials had high-risk bias for allocation concealment [20,25]. Two trials had unclear randomization without description in sufficient detail $[23,26]$. Five trials had lower risk bias for allocation concealment $[21,22,24,27,28]$. Three trials were doubleblinded trials with a lower risk for blinding participants and study personnel $[21,22,28]$. Two trials did not use a blinding method to participants and study personnel during the study with a high risk due to the great appearance difference between Chinese medical formulation pills and decoction with conventional pharmaceutics such as indomethacin, benzbromarone, and allopurinol [20, 25]. The other four trials had insufficient information to permit a judgment $[23,24,26,27]$. Three trials could be regarded as lower risk for blinding of outcome assessors [20-22]. One trial had high risk in outcome assessment due to a lack of blinding [25]. Five trials had unclear risk in outcome assessment without description in sufficient detail [23, 24, 26-28]. Two trials reported cases with poor compliance which were dropped from the study [22, 24], two trials reported cases lost to follow-up [21, 28], and two trials had missing values in the results $[20,27]$, which might affect the results. Four trials were considered as a high risk of attrition bias with selective data reporting $[21,23,27,28]$. The other five trials were classified as low risk [20, 22, 24-26]. For poor blinding and allocation design, one trial was assessed as a high risk of bias in the other resource [25]. Two trials were estimated at a lower risk because of their study design $[23,24]$. The other 


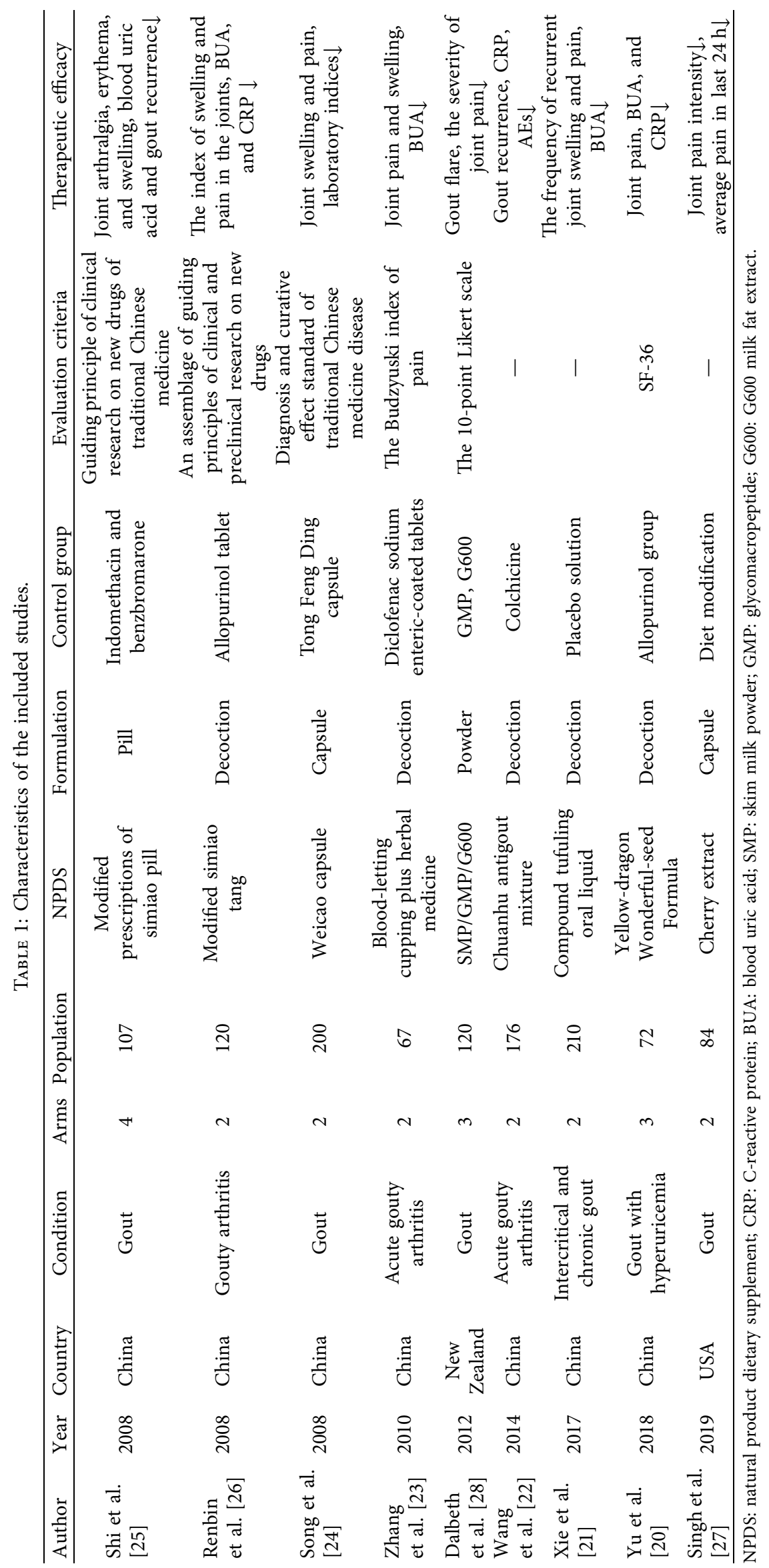


six were regarded as an unclear risk of other sources of bias [20-22, 26-28]. The methodological quality of the nine RCTs was poor because of two or more criteria listed as a high or unclear risk of bias. The study methodology was summarized in Figure 2.

\subsection{Measurable Outcomes}

3.3.1. Joint Swelling, Pain, and Limitation Relief. Study results suggested that NPDS treatments were superior to control interventions in joint swelling, pain, and activity limitation, except one trial which reported improvement just in joint pain-relieving, not in swelling and activity limitation [22]. In the double-blind RCT, 176 participants with acute gouty arthritis were divided to either the chuanhu antigout mixture $250 \mathrm{ml}$ orally daily plus placebo (mimetic agent of colchicine) or colchicine 1 pill $(0.5 \mathrm{mg} / \mathrm{pill})$ orally, twice daily, for 3 days and later once daily. All the participants orally administered pain-reliving medication etoricoxib $60 \mathrm{mg}$ once for 10 days. Results indicated that the chuanhu antigout mixture had a favorable effect in decreasing of joint pain score, instead of joint swelling and limitation compared with colchicine [22].

The evaluation criteria for therapeutic effect of joint swelling, pain, and activity limitation varied in the enrolled trials. Besides three trials $[21,22,27]$, the other six trials have different criteria to assess the therapeutic effect $[20,23-26,28]$. To investigate the compatibility of modified prescriptions of simiao pill on patients with acute gouty arthritis, Shi et al. [25] evaluated the clinical efficacy with "guiding principles of clinical research on new drugs of traditional Chinese medicine," which included 4 grades (none, mild, moderate, and severe), with scores $0,1,2$, and 3 , respectively. Affected joint arthralgia, erythema, and swelling, as well as blood uric acid level and gout recurrence were evaluated comprehensively. In the other trial to observe the efficacy of the modified simiao tang for gouty arthritis and blood uric acid, Renbin et al. [26] used "an assemblage of guiding principles of clinical and preclinical research on new drugs (western drugs)," which included "clinical cure, markedly improved, improved, and ineffective." The index of swelling and pain in the joints were scored according to the swelling and pain in the joints before and after treatment. Song et al. [24] classified the therapeutic effect in three grades: "markedly effective, effective, and ineffective" on the basis of the criteria "diagnosis and curative effect standards of traditional Chinese medicine disease." These criteria covered joint symptoms and signs as well as laboratory indices. Zhang et al. [23] used the criteria to assess efficacy effects with three categories of "cured, improved, and failed," based on both joint swelling and pain. The affected joint pain was assessed with the assessment of the Budzyuski index, which graded in $0,1,2,3,4$, and 5 with increasing pain severity. Dalbeth et al. [28] recorded gout flare and severity of pain with the 10-point Likert scale. Yu et al. [20] adopted the SF-36 scale to evaluate the change in affected joint swelling, pain and activity. The levels of SUA and urine urate were measured at week 0 and week 4 . A meta-analysis of joint swelling, pain, and limitation relief could not be employed due to the inconsistency and heterogeneity of interventions, controls, and outcome measures.

3.3.2. Serum Uric Acid (SUA) Level. Four RCTs included 420 patients (211 patients in the observation groups and 209 in the control groups) provided SUA changes before and after treatment. The study result showed that there was no significant difference between the NPDS interventions and the control groups in terms of SUA reduction (SMD $-1.80,95 \%$ CI: -4.45 to 0.86$)(p>0.05)$. Because $I^{2}=99 \%$, a randomeffect model was used for the analysis. Subgroups were divided by treatment; the subgroup meta-analysis showed that modified simiao tang was better in reducing SUA than the allopurinol tablet [26]. Due to the lack of detail in the reports, no meta-analysis was conducted in one-third of the trials [21-23, 28]. In addition, the other study reported serum urate value instead of SUA level [27] and was temporarily omitted from the meta-analysis. The effects of NPDS on the serum uric acid (SUA) levels of patients with gout are shown in Figure 3.

3.3.3. CRP Value. Four studies [20, 22, 26, 28] reported CRP value changes pre- and posttreatment, and the meta-analysis of three trials $[20,22,26]$ showed that the NPDS therapies were not more effective than treatment with conventional pharmaceutics such as allopurinol and colchicine $(N=232$; SMD, $-0.26 ; 95 \% \mathrm{CI},-0.55$ to 0.04$)(p>0.05)$. In the subgroup meta-analysis, modified simiao tang was superior to allopurinol tablets in decreasing CRP level [26]. The other study [28] which reported CRP change roughly was just temporarily omitted from the meta-analysis. The effects of NPDS therapies on CRP levels of patients with gout are shown in Figure 4.

3.3.4. Adverse Events. Five RCTs including 750 patients with 408 in the experimental groups and 342 in the control groups provided safety evaluation data [21, 22, 24, 27, 28]. The meta-analysis showed that NPDS therapies had not fewer adverse events than the control groups $(N=750 ; R R$, 0.47 ; 95\% CI, 0.20-1.11) ( $p>0.05)$. In total, 112 patients experienced side effects. In the subgroup meta-analysis, chuanhu antigout mixture and compound tufuling oralliquid had fewer AEs than colchicine and placebo solution separately $[21,22](p<0.05)$. The AEs included bitter taste, poor appetite, and leucopenia, as well as gastrointestinal reactions such as nausea, vomiting, diarrhea, and flatulence, which could be relieved by temporary dosage reducing or medication pause [22, 24, 28]. Xie and his colleagues [21] found fewer leucopenia incidence $(2.16 \%)$ in the treatment group of the compound tufuling oral liquid compared with that of the placebo solution $(9.86 \%)$, and more detailed information was not further reported. No other serious adverse events were recorded. Adverse events caused by NPDS interventions in patients with gout are shown in Figure 5. 


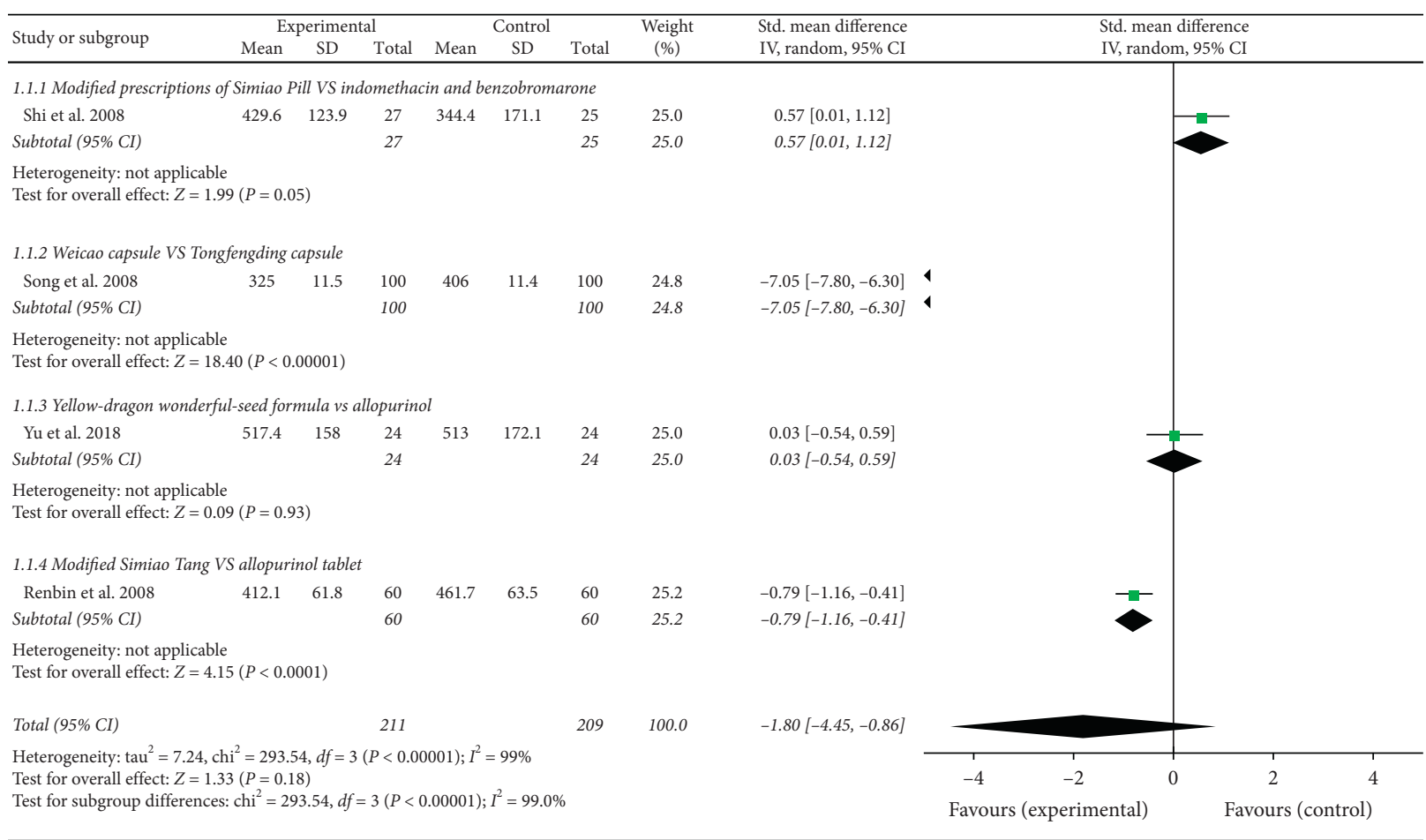

FIGURE 3: Effects of NPDS on the serum uric acid (SUA) levels of patients with gout.

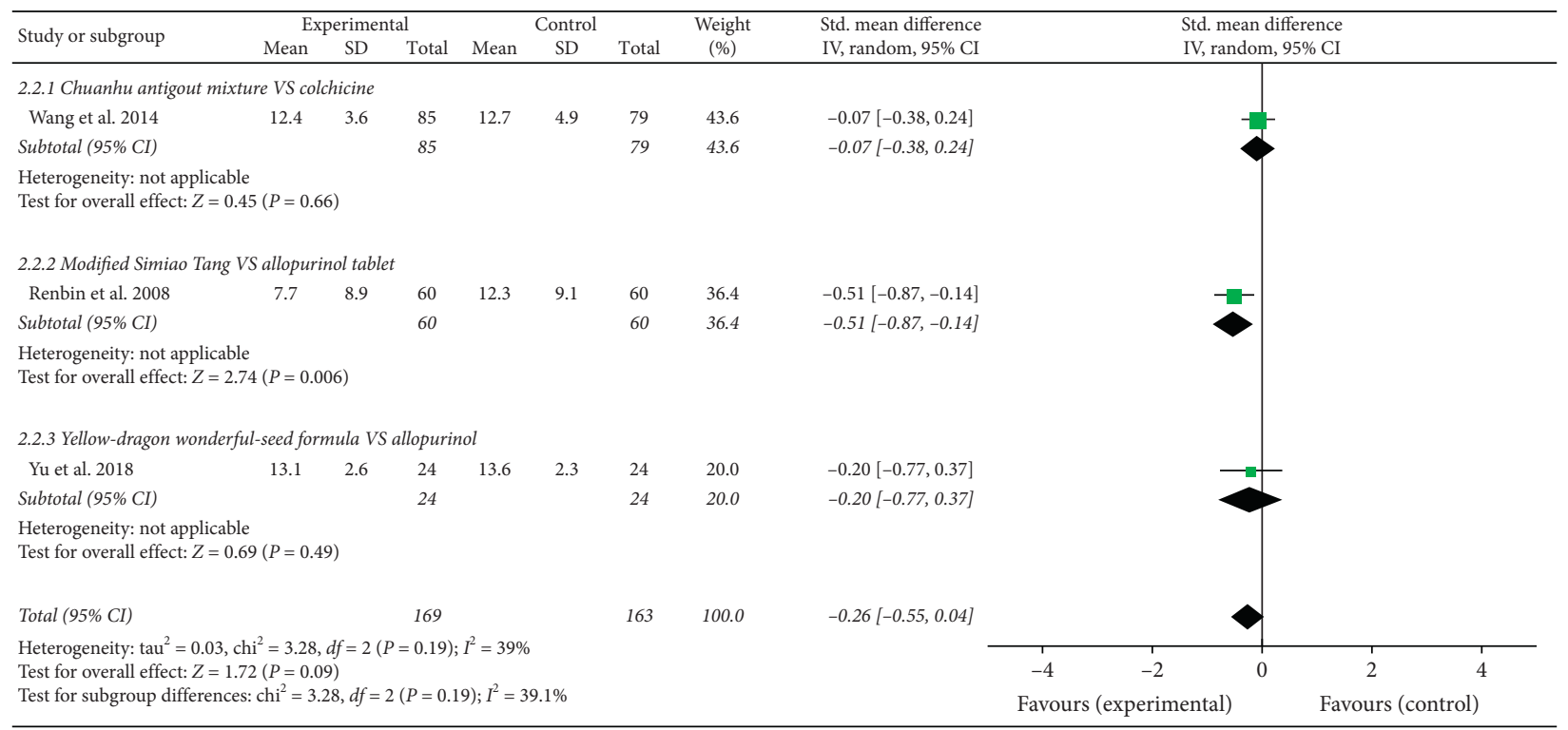

FIGURE 4: Effects of NPDS therapies on the CRP levels of patients with gout.

\section{Discussion}

Nine RCTs were included in our review, which compared Chinese herbs, cherry extract, and compound skim milk powder with a placebo control, conventional pharmaceutics, diet modification, and other Chinese medications for the treatment of gouty arthritis. Outcome measurements cover affected joint swelling, pain, and activity relieving, SUA and CRP value change, and AEs incidence.
The methodological quality of the included trials was poor with two or more assessments of high or unclear risk of bias. Despite mostly positive results in the therapeutic efficacy evaluation, the evidence data evaluating clinical efficacy were not convincing and robust, which could be attributed to the variety of evaluation criteria. Some studies comprehensively evaluated joint arthralgia, swelling, and activity limitation as well as laboratory indices [24, 25], some focused on joint swelling and pain [23, 26, 28], and one 


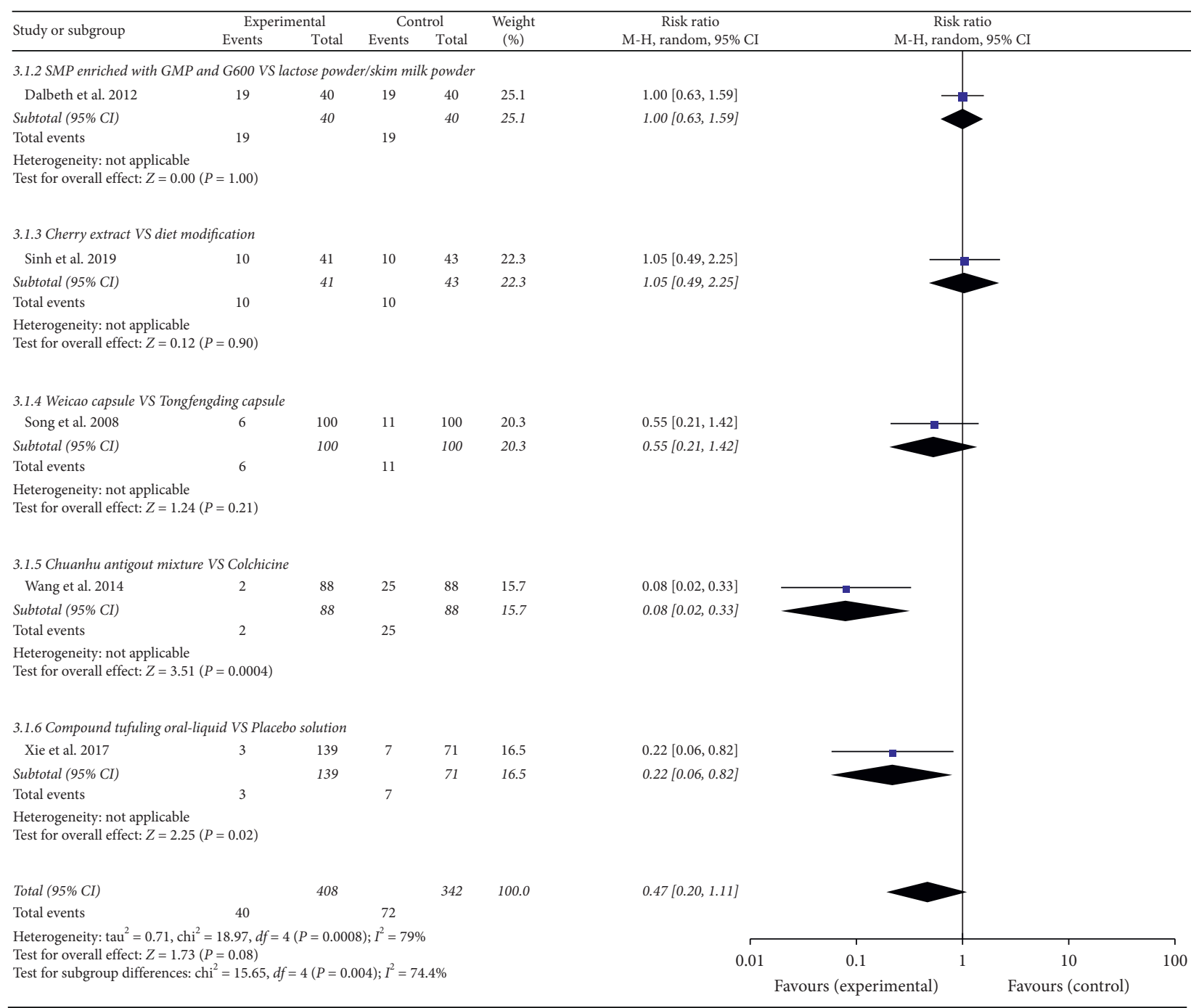

FIgURE 5: Adverse events caused by NPDS interventions in patients with gout.

utilized SF-36, SUA and urine urate for assessment [20]. Poor methodological quality and the small number of the included trials were the other contributors. Therefore, to fully assess the clinical role of NPDS in the treatment of gout, high-quality RCTs of standardized evaluation criteria with international recognition are required in the future.

Until now, nonsteroidal anti-inflammatory drugs (NSAIDs), colchicine, corticosteroids, and prophylaxis are guideline-recommended pharmaceuticals with highstrength evidence for individuals with gout [2], but the use of these drugs is often associated with unwanted side effects and adverse events [29]. NPDS interventions attract a great deal of public attention due to their perception as being nontoxic, natural, and economical properties [16]. Our review suggests that NPDS interventions may have some clinical efficacy in terms of symptom improvement, reduction in SUA and CRP levels, all with a lower level of adverse events compared with standard pharmacological treatment. They may possibly be associated with potential toxicity due to their multiherbal components and different formulations (e.g., decoctions and powders) with may be associated with gastrointestinal reactions.

Numerous articles have been written describing the important role and safety of NPDS therapies in patients with gout [15, 16, 30-34]; but until now, no review has quantitatively synthesized the relevant RCTs. To our knowledge, this review is the first overview of available RCTs investigating the efficacy and safety of NPDS approaches in gout management. This review highlights the impact of natural products on gout management and permits the identification of current evidence gaps, thus informing clinical decision making and guiding future research.

This review does have limitations. First, due to the study design limitation, we only searched RCTs published in English, thus possibly omitting important studies which appeared only in non-English journals. In particular, Chinese language journals may be an additional resource worthy of systematic review. Second, the included RCTs are of low quality. This makes the comparative analysis difficult to perform and reduces the confidence in the meta-analysis. 
Finally, the heterogeneity of the included studies was significant. More high-quality trials with large sample size RCTs are required in the future.

\section{Conclusion}

This review provides insight into the contemporary treatment of gout with NPDS. NPDS appeared to be superior to control groups in affected joint pain, swelling, and activity limitation, while not in decreasing SUA and CRP levels or the incidence of AEs. Current existing evidence is insufficient to permit a definitive statement about the efficacy and safety for gout patients due to poor trial quality and standardized evaluation criteria. Further larger and more rigorously designed RCTs are needed in the future.

\section{Conflicts of Interest}

The authors declare that they have no conflicts of interest.

\section{Acknowledgments}

Dr. Yang appreciates the Sanming Project of Medicine in Shenzhen, Shenzhen Nanshan People's Hospital, Guangdong Medical University, Shenzhen, China, and the support for her visiting study in Mayo Clinic. The authors thank the experienced librarian Leslie C. Hassett, Mayo Clinic Rochester Campus for the literature research of this review.

\section{References}

[1] G. Ruoff and N. L. Edwards, "Overview of serum uric acid treatment targets in gout: why less than $6 \mathrm{mg} / \mathrm{dL}$ ?," Postgraduate Medicine, vol. 128, no. 7, pp. 706-715, 2016.

[2] P. G. Shekelle, S. J. Newberry, J. D. FitzGerald et al., "Management of gout: a systematic review in support of an American college of physicians clinical practice guideline," Annals of Internal Medicine, vol. 166, no. 1, pp. 37-51, 2017.

[3] P. P. Khanna, G. Nuki, T. Bardin et al., "Tophi and frequent gout flares are associated with impairments to quality of life, productivity, and increased healthcare resource use: results from a cross-sectional survey," Health and Quality of Life Outcomes, vol. 10, no. 1, p. 117, 2012.

[4] Y. Zhu, B. J. Pandya, and H. K. Choi, "Prevalence of gout and hyperuricemia in the US general population: the national health and nutrition examination survey 2007-2008," Arthritis \& Rheumatism, vol. 63, no. 10, pp. 3136-3141, 2011.

[5] M. M. Elfishawi, N. Zleik, Z. Kvrgic et al., "The rising incidence of gout and the increasing burden of comorbidities: a population-based study over 20 years," The Journal of Rheumatology, vol. 45, no. 4, pp. 574-579, 2018.

[6] P. Richette, M. Doherty, E. Pascual et al., "2016 updated EULAR evidence-based recommendations for the management of gout," Annals of the Rheumatic Diseases, vol. 76, no. 1, pp. 29-42, 2017.

[7] M. Hui, A. Carr, S. Cameron et al., "The British society for rheumatology guideline for the management of gout," Rheumatology, vol. 56, no. 7, pp. e1-e20, 2017.

[8] I. Sari, S. Akar, B. Pakoz et al., "Hyperuricemia and its related factors in an urban population, Izmir, Turkey," Rheumatology International, vol. 29, no. 8, pp. 869-874, 2009.
[9] L. Qiu, X. Q. Cheng, J. Wu et al., "Prevalence of hyperuricemia and its related risk factors in healthy adults from Northern and Northeastern Chinese provinces," BMC Public Health, vol. 13, no. 1, p. 664, 2013.

[10] X. Liu, S. Huang, W. Xu et al., "Association of dietary patterns and hyperuricemia: a cross-sectional study of the Yi ethnic group in China," Food and Nutrition Research, vol. 62, no. $1380,2018$.

[11] J. C. DeSalvo, M. B. Skiba, C. L. Howe, K. E. Haiber, and J. L. Funk, "Natural product dietary supplement use by individuals with rheumatoid arthritis: a scoping review," Arthritis Care \& Research, vol. 71, no. 6, pp. 787-797, 2019.

[12] H. K. Choi, "A prescription for lifestyle change in patients with hyperuricemia and gout," Current Opinion in Rheumatology, vol. 22, no. 2, pp. 165-172, 2010.

[13] V. P. Gullo and D. E. Hughes, "Exploiting new approaches for natural product drug discovery in the biotechnology industry," Drug Discovery Today: Technologies, vol. 2, no. 3, pp. 281-286, 2005.

[14] D. M. Marcus, "Dietary supplements: What's in a name? What's in the bottle?," Drug Testing and Analysis, vol. 8, no. 34, pp. 410-412, 2016.

[15] S. Hao, C. Zhang, and H. Song, "Natural products improving hyperuricemia with hepatorenal dual effects," Evidence-Based Complementary and Alternative Medicine, vol. 2016, Article ID 7390504, 7 pages, 2016.

[16] W. H. El-Tantawy and A. Temraz, "Anti-fibrotic activity of natural products, herbal extracts and nutritional components for prevention of liver fibrosis: review," Archives of Physiology and Biochemistry, pp. 1-12, 2019.

[17] M. Andres, F. Sivera, L. Falzon, R. Buchbinder, and L. Carmona, "Dietary supplements for chronic gout," Cochrane Database of Systematic Reviews, vol. 10, Article ID CD010156, 2014.

[18] J. Savović, L. Weeks, J. A. Sterne et al., "Evaluation of the cochrane collaboration's tool for assessing the risk of bias in randomized trials: focus groups, online survey, proposed recommendations and their implementation," Systematic Reviews, vol. 3, no. 1, p. 37, 2014.

[19] J. P. T. Higgins, D. G. Altman, P. C. Gotzsche et al., "The cochrane collaboration's tool for assessing risk of bias in randomised trials," BMJ, vol. 343 , no. 2, Article ID d5928, 2011.

[20] X. N. Yu, H. Y. Wu, Y. P. Deng et al., “"Yellow-dragon wonderful-seed formula" for hyperuricemia in gout patients with dampness-heat pouring downward pattern: a pilot randomized controlled trial," Trials, vol. 19, no. 1, p. 551, 2018.

[21] Z. Xie, H. Wu, X. Jing et al., "Hypouricemic and arthritis relapse-reducing effects of compound tufuling oral-liquid in intercritical and chronic gout: a double-blind, placebo-controlled, multicenter randomized trial," Medicine, vol. 96, no. 11, Article ID e6315, 2017.

[22] Y. Wang, L. Wang, E. Li et al., "Chuanhu anti-gout mixture versus colchicine for acute gouty arthritis: a randomized, double-blind, double-dummy, non-inferiority trial," International Journal of Medical Sciences, vol. 11, no. 9, pp. 880885, 2014.

[23] S.-J. Zhang, J.-P. Liu, and K.-Q. He, “Treatment of acute gouty arthritis by blood-letting cupping plus herbal medicine," Journal of Traditional Chinese Medicine, vol. 30, no. 1, pp. 18-20, 2010.

[24] E.-F. Song, Q. Xiang, K.-M. Ren et al., "Clinical effect and action mechanism of Weicao capsule in treating gout," 
Chinese Journal of Integrative Medicine, vol. 14, no. 2, pp. 103-106, 2008.

[25] X.-D. Shi, G.-C. Li, Z.-X. Qian, Z.-Q. Jin, and Y. Song, "Randomized and controlled clinical study of modified prescriptions of Simiao Pill in the treatment of acute gouty arthritis," Chinese Journal of Integrative Medicine, vol. 14, no. 1, pp. 17-22, 2008.

[26] Q. Renbin, S. Ruizi, L. Dejiu, C. Yuanlin, and Y. Hongping, "Treatment of 60 cases of gouty arthritis with modified Simiao Tang," Journal of Traditional Chinese Medicine, vol. 28, no. 2, pp. 94-97, 2008.

[27] J. A. Singh, A. L. Willig, B. Darnell et al., "Patient-centered outcomes and key study procedure finalization in the pilot feasibility gout randomized trial: comparative feasibility study in gout, cherry extract versus diet modification (MiniGOUCH)," JCR: Journal of Clinical Rheumatology, vol. 12, p. 12, 2019.

[28] N. Dalbeth, R. Ames, G. D. Gamble et al., "Effects of skim milk powder enriched with glycomacropeptide and G600 milk fat extract on frequency of gout flares: a proof-of-concept randomised controlled trial," Annals of the Rheumatic Diseases, vol. 71, no. 6, pp. 929-934, 2012.

[29] R. K. Narang and N. Dalbeth, "Management of complex gout in clinical practice: update on therapeutic approaches," Best Practice and Research: Clinical Rheumatology, vol. 32, no. 6, pp. 813-834, 2018.

[30] Y. Zhang, T. Neogi, C. Chen, C. Chaisson, D. J. Hunter, and H. K. Choi, "Cherry consumption and decreased risk of recurrent gout attacks," Arthritis \& Rheumatism, vol. 64, no. 12, pp. 4004-4011, 2012.

[31] Y. Zhang, C. Chen, D. J. Hunter, C. E. Chaisson, H. K. Choi, and T. Neogi, "Cherry consumption and risk of recurrent gout attacks," Arthritis and Rheumatism, vol. 10, p. 1366, 2012.

[32] N. Corp and B. Pendry, "The role of western herbal medicine in the treatment of gout," Journal of Herbal Medicine, vol. 3, no. 4, pp. 157-170, 2013.

[33] R. N. Beyl, L. Hughes, and S. Morgan, "Update on importance of diet in gout," The American Journal of Medicine, vol. 129, no. 11, pp. 1153-1158, 2016.

[34] J. Lin, S. Chen, S. Li, M. Lu, Y. Li, and Y. Su, "Efficacy and safety of Chinese medicinal herbs for the treatment of hyperuricemia: a systematic review and meta-analysis," Evidence-Based Complementary and Alternative Medicine, vol. 2016, Article ID 2146204, 12 pages, 2016. 